\title{
Information Services Model based on Publish/Subscribe for Large Scale Sensor Networks
}

\author{
Biao Dong ${ }^{1, a}$, Jinhui Chen ${ }^{2, b}$ \\ ${ }^{1}$ School of Computer \& Software, Nanjing Institute of Industry Technology, Nanjing, 210023, China \\ ${ }^{2}$ School of Computer \& Software, Nanjing University of Information Science \& Technology, Nanjing, \\ 210044, China \\ aemail:dongb@niit.edu.cn, bemail:cjh@nuist.edu.cn
}

Keywords: Information Service; Publish/Subscribe; Sensor Networks

\begin{abstract}
This paper presented an approach for modeling and implementing the architecture of large scale sensor networks(LSSNs) information services applications using publish/subscribe (Pub/Sub) paradigm. Considering the high level abstraction and the dynamic characteristics which were necessary for large-scale deployment, information service resource view was defined to describe the functional relationships between information services, and a P2P-based information service discovery mechanism was constructed to detect information services. The analysis indicated that the information services model provided sensing-based service management and dynamic configuration management for LSSNs.
\end{abstract}

\section{Introduction}

Information service is a kind of network component which has the characteristics of autonomy, openness, self-description, and implementation-independent. Information service computing uses services as basic elements to develop the distributed application programs[1]. Information services are playing more and more important role in LSSNs: Information services provide complete solutions for users by integrating information services and data into LSSNs, and are more focused on their own functions. In the traditional Internet, the service invocation based on service-oriented architecture occupies a dominant position in system architecture. In LSSNs, sensing-based service systems play more important roles. Since event-driven architecture can integrate sensing and service, it has been the dominating architecture in LSSNs information services.

$\mathrm{Pub} / \mathrm{Sub}$ is an asynchronous communication paradigm that supports many-to-many interactions between a set of clients. A client can be an information publisher, an information subscriber, or both. Client interactions are data-centric: publishers describe their publishable events, subscribers express their interest in events, and the Pub/Sub protocol delivers the published events to their corresponding event subscribers. The loose coupling of clients eliminates the burden of context information gathering and processing by resource constrained devices and also supports reuse of context information. In addition, it hides context information access and low-level sensor operations from applications, and therefore allows dynamic reconfiguration of the set of sensors providing context information to the applications[2]. The Pub/Sub protocol is considered as a way to achieve large-scale distributed event-driven mechanism, so this paradigm is a suitable paradigm for LSSNs information services.

Sarakis presented virtual sensor networking. The concept of virtual sensor networking is an approach that enables the decoupling of the physical sensor deployment from the applications running on top of it, helping the proliferation of new services and applications beyond the scope of the original deployment[3]. Seeger presented an event-driven middleware for on-body and ambient sensor networks that allows multiple applications to define information types of their interest in a publish/subscribe manner[4]. Radianti proposed a smartphone based communication framework for disaster specific machine learning techniques. Core to the framework is a robust content-based publish-subscribe mechanism that allows flexible sharing of sensor data and computation results[5]. Perera provided a comprehensive overview of the sensing as a service model and its applicability, 
discussed the model from three different perspectives including technological, economical and social, and examined how the sensing as a service can be a sustainable, scalable and powerful model[6]. Byun proposed an open source platform which can collect and share event data for a broad range of Internet of things applications, and discussed a scalable and flexible architecture, the document-based event model[7].

The above researches effectively improve the efficiency of information system development for LSSNs, but a key problem of LSSNs information service for further study is how to provide high level abstraction in order to solve problem of large-scale deployment. This paper proposes an information service architecture for designing and developing LSSNs information systems. Our architecture supports the following features:

(1) Integrated information sercices with Pub/Sub.

(2) In terms of technical implementation, we use an information service resource view to describe the functional relationships between information services.

(3) P2P-based information service for dynamic LSSNs.

\section{Server-side Information Service Architecture}

The information service system is composed of client-side runtime library and server-side infrastructure. Client-side runtime library provides Pub/Sub interfaces for the upper applications, shields the clients from the complexity of communication protocol. The server-side infrastructure is composed of management nodes and proxy nodes. The management node is responsible for client access, cluster partitioning, node joining and topology management. In addition to completing the three basic functions, such as topology management, subscription management, notification message routing, the proxy node also considers the high availability of system, the dynamic migration of service, the effective utilization of system resources, and efficient event-distributed mechanism. Fig.1 shows the integrated server-side information service architecture when Pub/Sub is used as middleware. The framework includes information service layer and protocol control layer.

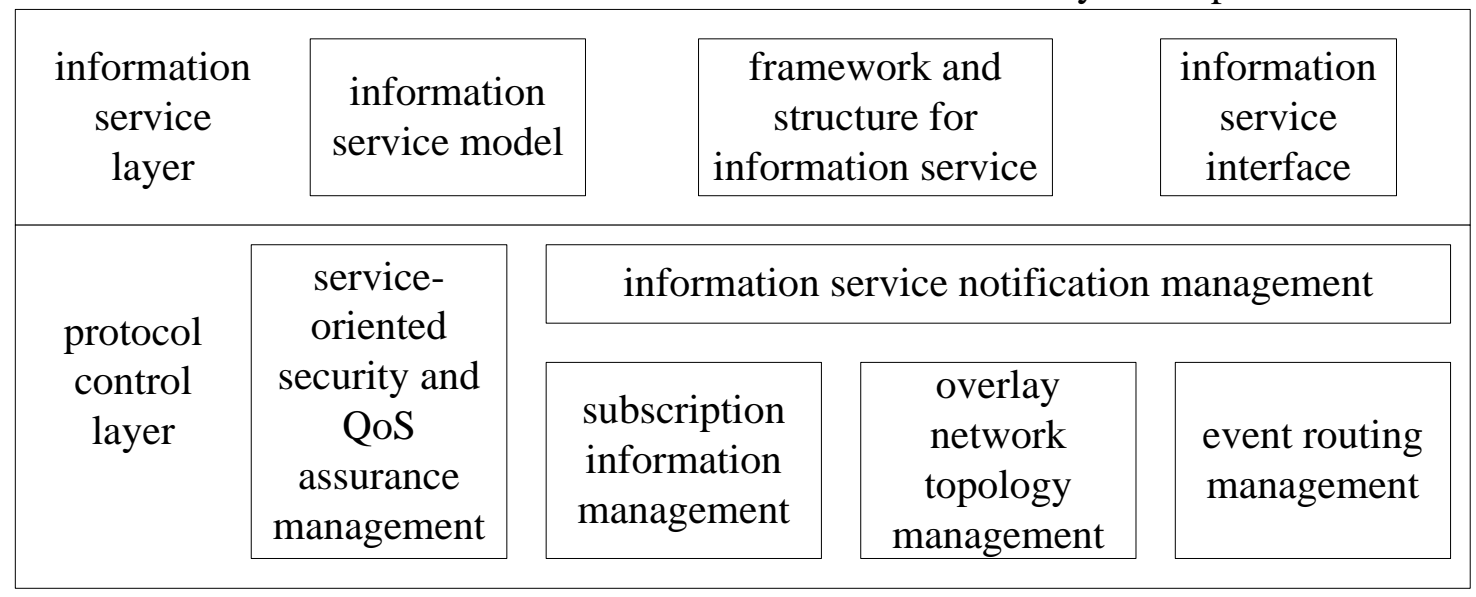

Fig.1. Server-side information service architecture with Pub/Sub

The information service layer includes an information service model, a framework and structure for information service and an information service interface. The information service interface completes the communication and interaction between the users and the system. It is used to enter the user's needs and return the desired service results.

The protocol control layer consists of the following modules, such as service oriented security and QoS assurance management, information service notification management, subscription information management, overlay network topology management and event routing management. Their functions are described as follows.

This information service notification management module is responsible for registration management, event notification and event cache management. According to the update of the client subscription table, the subscription information management module calculates whether to update the subscription information of the proxy node. By summarizing the subscription update message 
for the proxy nodes in the cluster, the module updates the proxy node subscription table, and calculates whether the subscription information for the cluster needs to be updated. For a representative node, the module maintains a clustered subscription table by summarizing the subscription updating messages for each cluster. Together with management nodes, the overlay network topology management module is responsible for the processes of node joining, exiting and failure, and assists the subscription information management module in realizing the intra and inter-cluster subscription messages forwarding. The event routing management module is responsible for event routing. Namely, this means using subscription table information to calculates the routing table, and forwarding data in event buffer pool according to the routing table.

\section{Implementation Technology of Information Service}

In the information service layer, we provide the users with well-organized service resources at the application level through organizing, classifying and managing service resources in LSSNs. And on the basis of the service resources, discovery and integration of user required services are completed further. The key technologies that related to the implement of information service includes the service function clustering methods, the mechanism of service resource management, the mechanism of service integration.

The service function clustering method merges the multiple service instances of the same service in service instance set, and analyzes the association and dependency relation of the different services. From a large number of discrete, loosely-coupled service resources, the method analyzes and identifies a set of characteristics which embodies the essence of the service. The method provides an abstract service subset which is easy to be understood and used by the users. In the field of software engineering, the faceted classification method is widely used for software component classification and management. Its main idea is to accurately classify the components by facets. The facet classification pattern is more objective and comprehensive, and its scalability is suitable for LSSNs. So, according to the faceted classification schema, we cluster and abstract the information service resources in application semantics.

According to the structure of multi-faceted directory tree, information service resources form a network structure by means of clustering analysis. The network is the basis of the service resource management. The service needs of users often require a combination of multiple simple services according to certain rules. In order to better support service integration, we use an information service resource view to further describe the functional relationships between information services. This view forms a service relation network with hierarchical structure. It consists of the following three parts, such as a set of abstract services, the relationships between services, and interactive message sequences which are generated when the services are integrated. The service resource management supports the discovery and integration of services through the reasoning mechanism which allows us to more easily and effectively manage service resources view.

From a large number of service resources, the service integration find the required services and the service compositions, assembles new services, and completes service or computing functions that users request. These processing activities, such as query, selection and assembly of service resources, are in service component level. Generally, the demands of users are in the service and computation level. In order to establish the relationship between the user needs and the processing of service components, we propose a method to describe the user's requirements based on hierarchical processing model. In this model, the highest level describes the classifications and functions of services; the second layer describes functional decomposition strategies of services; the third layer includes a variety of service integration solutions corresponding to the functional decomposition strategies.

\section{P2P-based Information Service for Dynamic LSSNs}

The particularities of dynamic LSSNs information service are reflected as follows. First, information providers and even information services themselves frequently reach or leave the 
network, so the information service should be scalable. Second, information services on dynamic LSSNs should be lightweight. Third, information services on dynamic LSSNs can automatically detect the presence of information providers. Correspondingly, information service providers can also find the active information services. Finally, standard interfaces should be adopted to integrate heterogeneous networks. We propose a P2P-based information service mechanism which is lightweight and dispersive. Information services are independent. At the same time, they're also in collaboration with each other. For information service users and information providers, information services are dynamic.

P2P-based information service discovery mechanism plays an important role in the information service system of dynamic LSSNs. Each information service is a peer entity which connects with other information services, and generates and manages a peer entity list. In order to establish and update the list of peer entities, an information service finds other information services by broadcasting messages. The information service that has received broadcast message sends a message to confirm it. Each information service has an endpoint reference(EPR) by which information services are distinguished from each other, and returns an EPR to sender in the confirmation message. In dynamic LSSNs, packets are easy to be lost due to channel errors, changes of routing, and broken connection. Considering the above factors, we adopt the following methods. A peer only sends broadcasting messages to its neighbor nodes. And then, these nodes forward the broadcasting messages to their neighboring nodes. Service discovery algorithm which is designed according to this method can greatly reduce packet losses.

Information services have dynamic characteristics. When a peer arrives at a network, it doesn't know what information services exist. In order to avoid the broadcast storm, the peer broadcast messages to the adjacent nodes, which has one hop distance away from the peer, in order to detect information services. If there is an information service which is adjacent to the peer, then the detection process is over. The peer gets an EPR. If all adjacent peers don't provide the information service, the peer sends another message to peers with two hops distance. This process continues until the discovery of information service. After information service has been successfully detected, the peer uses subscription mechanism to obtain resource information from the information service provider. When information service leaves network or fails, the peer will detect this change, and restart discovery process. Similarly, information sources have the same dynamic mechanism. When information source reaches network, it also doesn't know which information service can be registered. First, by using the same method as a peer to detect information services, information source obtains the latest information service EPR. Then, the information source uses subscription mechanism to update its status.

\section{Conclusion}

In this paper, we propose an approach for modeling and implementing the architecture of LSSNs information services applications using Pub/Sub paradigm. We derive conclusions of the information services model from analysis. The results show that the model provides sensing-based service management and dynamic configuration management for LSSNs, and easily be constructed.

\section{Acknowledgement}

This work was sponsored by Qing Lan project(Jiangsu province, china).

\section{References}

[1] J.Gubbi, R.Buyya, S.Marusic, et al. Internet of Things (IoT): A vision, architectural elements, and future directions[J]. Future Generation Computer Systems, 29(7), 2013, pp.1645-1660.

[2] B.Dong. Reconfigurable Publish/Subscribe Middleware for Wireless Sensor Networks[C]. Applied Mechanics and Materials, 2014, 536: 740-743. 
[3] L.Sarakis, T.Zahariadis, H.C.Leligou, et al. A framework for service provisioning in virtual sensor networks[J]. EURASIP Journal on Wireless Communications and Networking, 2012(1), 2012, pp.1-19.

[4] C.Seeger, K.Van Laerhoven, J.Sauer, et al. A Publish/Subscribe Middleware for Body and Ambient Sensor Networks that Mediates between Sensors and Applications[C]. Healthcare Informatics 2013 IEEE International Conference, 2013, pp.199-208.

[5] J.Radianti, J.J.Gonzalez, O.C.Granmo. Publish-subscribe smartphone sensing platform for the acute phase of a disaster: A framework for emergency management support[C]. Pervasive Computing and Communications Workshops(PERCOM Workshops), 2014, pp.285-290.

[6] C.Perera, A.Zaslavsky, P.Christen, et al. Sensing as a service model for smart cities supported by internet of things[J]. Transactions on Emerging Telecommunications Technologies, 25(1), 2014, pp.81-93.

[7] J.Byun, D.Kim. Oliot EPCIS: New EPC information service and challenges towards the Internet of Things[C]. RFID 2015 IEEE International Conference on IEEE, 2015, pp.70-77. 